\title{
Extraction of Coastal Ocean Wave Fields From SAR Images
}

\author{
Fabrice Collard, Fabrice Ardhuin, and Bertrand Chapron
}

\begin{abstract}
Wave spectra derived from synthetic aperture radar (SAR) images acquired by ENVISATs are compared to in situ measurements by seven sensors, deployed in a field experiment carried out on the French coast of La Manche (English Channel). The wave spectra inversion scheme is adapted for shallow water from the European Space Agency (ESA)'s operational processing techniques used for level 2 ocean wave products. Under the low to moderate wind speed observed conditions, overall good agreement is found between in situ and SAR observations over a wide range of wave heights and directions, including waves propagating in the radar azimuth direction and incidence angles different from the standard imagette products.
\end{abstract}

Index Terms-Ocean waves, synthetic aperture radar.

\section{INTRODUCTION}

$\mathbf{K}$ NOWLEDGE of wave conditions, either as climatology or as short-term forecast, is critical for all human activities at sea, including shipping, fishing, oil extraction, and naval operations. The development of wave models has been very fruitful over the past few decades, and wave forecasts are now quite reliable in the open ocean. Because wave models compute the wave field from surface winds, often provided by atmospheric models, this progress was made possible by advances in weather forecasting and remote sensing of surface winds over the oceans [1]. This reliability of wave models in forecasting significant wave heights has also been established thanks to wave height measurements from space-borne range altimeters as well as in situ observation from wave buoys. Current efforts to improve global wave forecasting are now limited by the poor availability of spectral wave measurements that may validate choices in the model parameterizations that are tuned to give similar wave heights on average, but often result in widely different spectral shapes. In particular, it is widely recognized that long period waves unrelated to local winds (swells) are still poorly predicted [2]. Synthetic aperture radar (SAR) "imagettes" covering small areas of the ocean surface $(10 \times 5 \mathrm{~km})$ have now been acquired routinely since 1992 by ERS-1 and -2 and now ENVISAT. These data are still little used because of limitations in the conditions under which a wave spectrum can be easily retrieved from the radar image and the difficulty of assimilating

Manuscript received October 8, 2004; revised March 21, 2005; accepted May 31, 2005. Associate Editor: R. Romeiser.

F. Collard is with BOOST Technologies, 29280 Plouzané, France (e-mail: fabrice.collard@boost-technologies.com).

F. Ardhuin is with SHOM, 29200 Brest, France (e-mail: ardhuin@ shom.fr).

B. Chapron is with CERSAT-IFREMER, Techn. Brest-Iroise, 29200 Brest,

France (e-mail: bertrand.chapron@ifremer.fr).

Digital Object Identifier 10.1109/JOE.2005.857503 wave spectra with variable azimuth wavenumber cutoff. Furthermore, the emphasis on significant wave height in the definition of the "analyzed wave field" at forecasting centers [1], relative to other variables such as swell wave height or peak period, has undervalued the long lasting impact of a proper SAR assimilation [3]. At the same time, the usefulness of data assimilation has been reduced by dramatic improvements in global wave forecasting of wind sea waves over the past decade [1].

Yet, the processing of SAR images has progressed, and, surprisingly, the use of SAR images for ocean waves in coastal areas has been extremely limited in spite of recognized larger model errors [3], resulting in poorer knowledge of local wave conditions. SARs, and in particular ENVISAT's advanced SAR (ASAR), offer a unique potential for imaging large areas, providing information about the spatial variations of the wave field. Indeed, because SAR measurements are generally limited but well adapted to the measurements of very long waves, SAR images generally reveal details on the swell transformation that are related to the underlying bathymetry and/or coastal currents [4], [5].

Hereafter, the extension to coastal areas of an algorithm designed for wave spectral estimation from single look complex (SLC) SAR images is described. The performance of this algorithm is discussed for different incidence angles, with a first validation with in situ data.

\section{WAVE MEASUREMENT BY SAR}

\section{A. Inversion Principles}

SARs rely on the displacement of the antenna (e.g., with a satellite) to provide a fine resolution in the flight (azimuth) direction that could only be achieved by a fixed antenna with a much larger aperture. The precise determination of the origin of echoes in the azimuth direction is given by the Doppler shift of the scattered signal. Resolution in the other (range) direction is given by the usual range gating of the return signal. Therefore, the echo of a fixed scatterer at the ocean surface can be transformed into a pixel on the SAR image. This image is formed by mapping the intensity of the backscattered signal to physical space, assuming that scatterers are not moving. Because the ocean surface does move, the SAR image will show the scatterers not at their true position in physical $(x-y)$ space, but rather at their position in "range-Doppler" space. Over the ocean, the electromagnetic (EM) waves are scattered with a mean power that is related to the roughness of the surface at the scales comparable to the EM wavelength $\lambda$ and modulated by the slope of the ocean surface over scales much larger than 


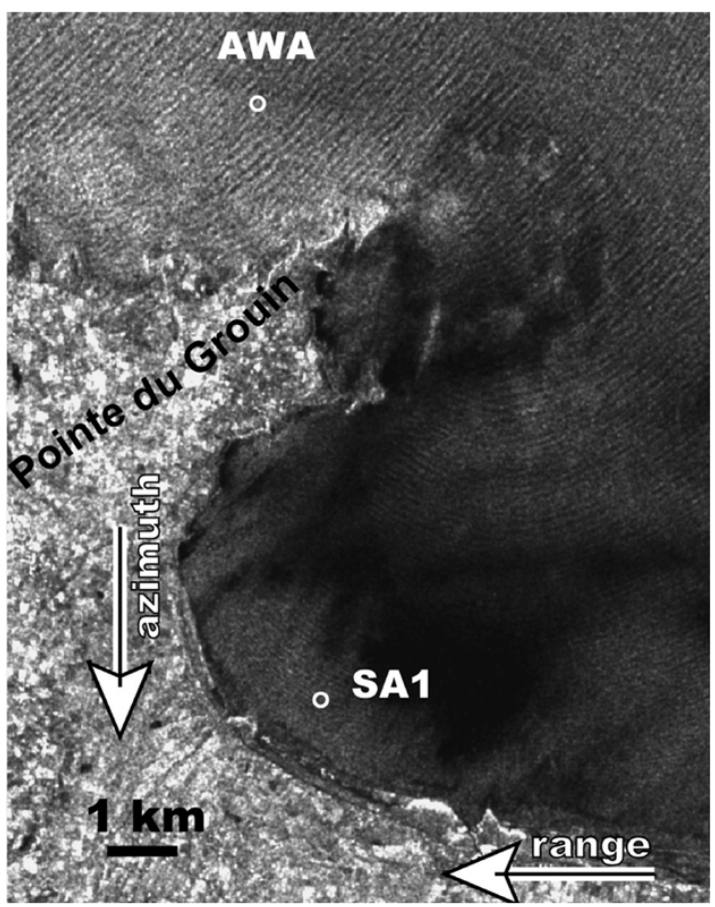

Fig. 1. Excerpt of the image acquired by ENVISAT on March 9, 2003 at $21 \mathrm{~h}$ $45 \mathrm{~min}$. The position of two instruments is indicated (AWA and SA1). The dark areas correspond to regions of very weak winds. The refraction of waves around the Pointe du Grouin is clearly visible.

$\lambda$. At C-band $\lambda \approx 5 \mathrm{~cm}$, ocean waves are visible thanks to three main imaging processes:

- the wind sea and swell modulate the amplitude of the shorter waves, and thus the backscatter intensity (hydrodynamic/aerodynamic modulation);

- the wind sea and swell modulate the local geometry and slopes of the sea surface (tilt modulation);

the wave orbital velocities, with surface convergence and divergence, produce modulations in the image as the scatterers moving on the waves are placed according to these induced Doppler velocities. This process is known as "velocity-bunching" and is mostly due to the wind sea (shorter and steeper waves).

The wavy pattern on SAR images [e.g., Figs. 1 and 2(a)] is generally described by its two-dimensional (2-D) wavenumber spectrum [e.g., Fig. 2(b)], which, after inversion, is related to the ocean surface elevation variance (wave) spectrum [e.g., Fig. 3(a)]. After many developments, a certain consensus has been reached on the SAR imaging mechanisms of the ocean surface waves, and analytical formulations are available for simulations of SAR spectra from real ocean wave spectra [6]. For space-borne measurements, velocity bunching is recognized to be a dominant modulation mechanism for waves with crests almost aligned with the range direction, and it is well described by theory. Hydrodynamic and aerodynamic modulation theories still lack satisfactory descriptions for scatterer distribution over longer wave profiles [7], and it will be neglected here. For low incidence angles, the tilt modulation is found to dominate for range-traveling waves.

Based on the theoretical nonlinear relation giving SAR spectra from wave spectra, different algorithms have been developed for inverting wave spectra from SAR spectra. A retrieval algorithm generally attempts to reconstruct the ocean wave spectrum by minimizing the difference between its corresponding theoretically mapped SAR spectrum (obtained with the forward transformation) and the satellite observation. The exact derivation of the nonlinear transform being too cumbersome to carry out, most of the inversion schemes partially ignore the complete nonlinear mapping and mostly use the simplifying gradient of a so-called optimized SAR quasi-linear transform that best matches the full nonlinear transform [8], [9]. Improvements to this technique have then focused on a better partitioning of the first guess wave spectral information [10].

The present paper follows an approach that avoids the need for a first guess for the wave spectrum. The entire wave spectrum is estimated from the SAR image alone. This type of inversion is done in two steps: one for the wind sea part of the spectrum, the other for the swell. Swell-induced motions usually result in a constructive imaging mechanism, while most of the blurring destructive effects are associated to the unresolved motions induced by the shorter and steeper wind sea.

For ENVISAT wave mode products, the first step uses the complete nonlinear SAR forward transform starting from an a priori knowledge of the wind speed and related wind sea spectrum [11]. Subsequently, the two main parameters that govern the wind sea (its degree of development and mean direction) are optimized. The a priori wind sea spectrum is thus adjusted, starting from a local wind vector to match the higher wavenumber nonlinear part of the measured SAR spectrum. The wind vector can often be determined from the image itself, and otherwise must be obtained from other measurement or numerical model output. In the second step, the swell spectrum is directly estimated from the residual signal, i.e., the observed SAR spectrum minus the nonlinear wind sea contribution evaluated in step 1 . To further simplify the inversion, step 2 is usually done by assuming linear mapping, i.e., linear modulation transfer functions (MTFs) between the surface elevation and the SAR image.

To ensure the validity of the inversion, this two-step scheme is iterated to check the consistency between retrieved swell spectra and observed low-pass filtering effects. Because complex images are now available, the $180^{\circ}$ ambiguity in the swell propagation direction can be removed by the analysis of cross-spectra images between different looks formed from the same image [12]. These different looks correspond to successive views of the same scene with short time lags. This technique takes advantage of the finite integration time of the sensor. On ENVISAT, one wave is observed continuously over $0.7 \mathrm{~s}$, and its motion during that time can be detected.

\section{B. Description of the Algorithm}

The algorithm used here is an extension of the algorithm used to produce level 2 wave spectra products from the European Space Agency (ESA)'s ENVISAT ASAR wave mode products. In practice, the processing of one image block (or one wave mode product) involves the following operations.

(i) Wind speed is estimated from the measured radar cross section (i.e., the image must be calibrated) and an 

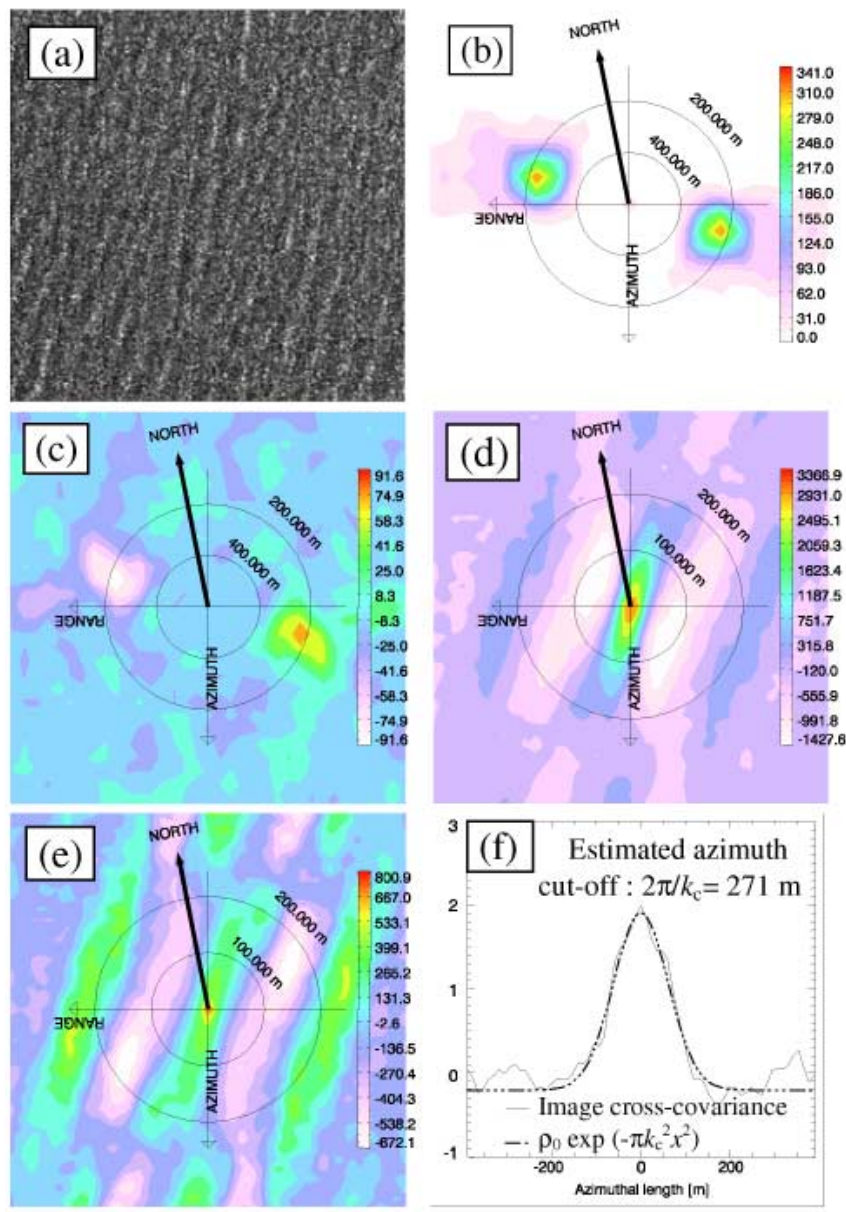

Fig. 2. Illustration of the steps in the wave spectra inversion for the imagette located near the DW1 instrument (Fig. 5). (a) Intensity image. (b) Image spectrum. (c) Imaginary part of the image cross-spectrum used for direction ambiguity removal. (d) Autocovariance of the image. (e) Enhanced image autocovariance after removal of nonlinear contribution. (f) Azimuth cutoff used for quasi-linear spectrum correction.
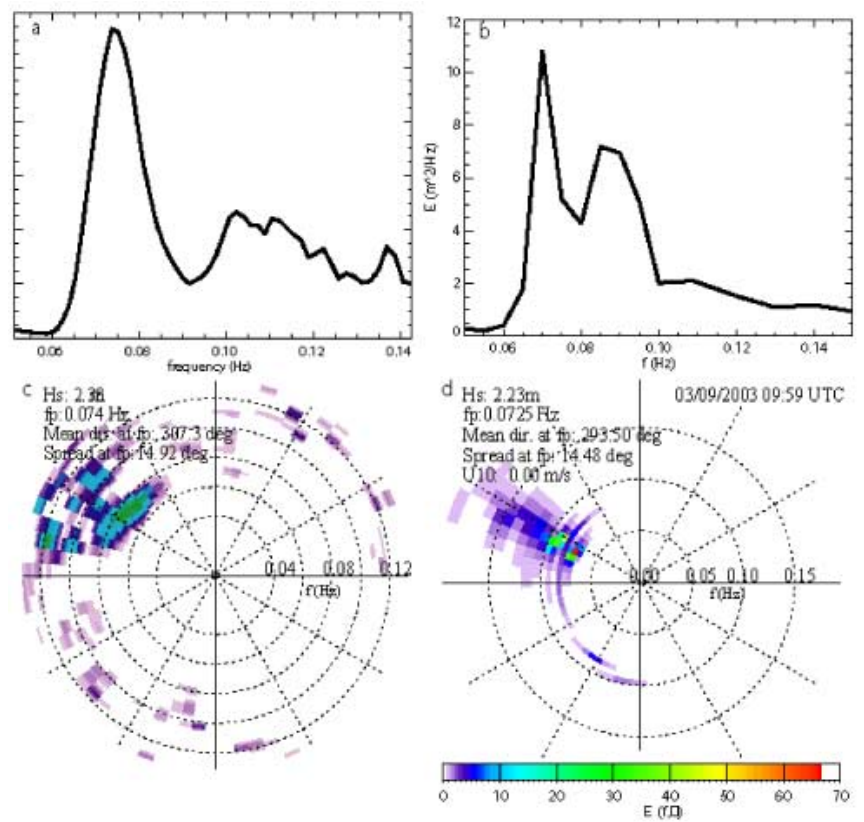

Fig. 3. (a) and (c) Example SAR-derived and (b) and (d) buoy-derived frequency spectrum and frequency-direction at DW $1\left(\theta_{I}=37^{\circ}\right)$. Because the graph stops at $f=0.14 \mathrm{~Hz}$, only swell waves are shown. 
a priori wind direction, or visual inspection/ low-wavenumber analysis of the image, using the backscatter model function (CMOD) described in [13].

(ii) The input SLC image [Fig. 2(a)] is detrended using a Gaussian low pass filter where the width of the filter is set such as to best remove the lower frequencies not related to waves.

(iii) Spectra and cross-spectra are computed corresponding to three extracted looks with time lags of $-0.27,0$, and $+0.27 \mathrm{~s}$. An a priori estimation of the speckle noise is used to reduce the variance of the spectra. A weighted sum of the spectra and cross-spectra then provides one single estimation of the spectrum [Fig. 2(b)] and the imaginary part of the cross-spectrum [Fig. 2(c)]. The weights given to each spectrum are functions of the image clutter noise level estimated from the high-frequency energy in the cross-spectrum.

(iv) Spectra are corrected for nonlinear SAR effects. For deep water and $23^{\circ}$ incidence, which is ENVISAT's wave mode configuration, this is done with look-up tables based on direct simulations. Here, this operation is performed with a resampling of the image crosscovariance function at short lags [Fig. 2(d) and (e), see Appendix].

(v) The azimuth cutoff wavenumber $k_{c}$ is estimated by fitting a Gaussian function to the autocovariance function of the cross-spectrum image for zero range lag [Fig. 2(f)], and the image spectrum (after step 4) is multiplied by a factor $\exp \left(k_{x}^{2} / k_{c}^{2}\right)$, where $k_{x}$ is the azimuthal wavenumber, that corrects for the cutoff effect. This correction is not applied to the highest frequency part of the spectrum.

(vi) The linear system transfer functions are estimated and applied (instrument transfer function and image to wave elevation MTFs), giving a symmetric surface elevation wavenumber spectrum in which the wave direction is only known with a $180^{\circ}$ ambiguity.

(vii) In order to remove this ambiguity, the signal-to-noise ratio (SNR) of the asymmetric spectra is estimated as the cross-spectral level of the resolved wave motion normalized by the spectral level in the high wavenumber part of the cross-spectrum. If this SNR is above a certain threshold, then the asymmetric spectrum is smoothed and the final symmetric and asymmetric wave spectra are obtained from a combination using their clutter noise levels to yield the ambiguity-free wave spectrum [Fig. 3(c)]. If the SNR is below a certain threshold, no ambiguity removal is performed and the symmetric spectrum is kept unchanged.

(viii) Although SAR spectra are originally wavenumber spectra, in situ observations at fixed positions yield frequency spectra. SAR spectra were thus transformed to frequency-directional spectra using the general dispersion relation. Effects of stronger nonlinearity and changes in the hydrodynamic MTF may impact the SAR image analysis but are not considered here. The inversion algorithm has not been tuned in any way to the observations.
As described, this inversion procedure primarily estimates the wave spectrum resolved by SAR (the detected wavy pattern in the SAR scene). It is thus mainly adapted to long wave systems. Note that, although the extraction of the swell is based on the quasi-linear transform, the inversion uses the full theoretical nonlinear SAR mapping through the coupling with the nonlinear part. This algorithm was developed originally for the wave inversion of ENVISAT ASAR wave mode at a unique incidence angle of $23^{\circ}$, explicitly assuming that observed waves are in deep water. The SAR products tested here are SLC image mode products with incidence varying from $15^{\circ}$ to $40^{\circ}$. These images cover coastal areas where the deep water assumption is no longer valid. The inversion algorithm has therefore been generalized in two ways.

First, the SAR MTF accounting for short wave tilted by larger waves has been generalized to take into account the local variation of backscatter around the changing incidence angle (different from $23^{\circ}$ ). This is simply implemented by applying for all incidence angles the CMOD empirical relationship relating incidence to C-band backscatter [13]. Second, the surface orbital velocities for a given wavenumber, entering the velocity bunching MTF, have been modified to take into account the linear dispersion relation for surface gravity waves $\omega^{2}=g k \tanh (k h)$, applicable to any water depth $h$, with $g$ as the acceleration of gravity and $k$ and $\omega$ as the wavenumber and radian frequency of the waves, respectively. This replaces the deep water limit $\omega^{2}=g k$. As a result, the extra parameter $h$ must be found. Here, $h$ is obtained from hydrographic soundings (Fig. 4).

Finally, the main modification is related to the fact that $a$ priori look-up tables were not available for incidence angles different from $23^{\circ}$ in order to evaluate nonlinear SAR mapping at different wind speeds and directions. It is well known that close to its origin, the autocovariance function of the SAR image is generally much broader than the corresponding real aperture radar (RAR) image, and the same thing is true for the cross-covariance of multiple looks. This blurring of short scales is related to surface nonlinearities including velocity bunching. Rather than extracting the nonlinear spectral part in the spectral domain, the auto- and cross-covariance functions have thus been resampled along their spatial coordinates to minimize the random blurring effects associated with the surface motions. This deconvolution procedure follows the theoretical mapping between SAR and RAR pixels (see Appendix).

\section{VALIDATION OF SAR-INVERTED SPECTRA IN COASTAL AREAS}

Observations were obtained as part of the "EPEL-2003" experiment initially designed for the validation of wave models in strong tidal environments (organized by SHOM and CETMEF). These measurements coincided with the deployment of other instruments on the tidal flats of the Baie du Mont Saint Michel by Ifremer and the University of Caen, where the hydrodynamics and sediment dynamics are under investigation in relation to the evolution of oyster and mussel farming. The area where wave measurements were performed is under the influence of North 
Atlantic swells and strong tides (typical tidal range is $10 \mathrm{~m}$, with currents often over $1 \mathrm{~m} / \mathrm{s}$ ).

A total of seven ASAR SLC products were delivered by ESA, corresponding to images acquired on February 24, and March 6 and 9. Out of these, only three contained good wave information and were acquired on March 9 at $10 \mathrm{~h} 22 \mathrm{~min}, 10 \mathrm{~h} 23 \mathrm{~min}$, and $21 \mathrm{~h} 44$ min coordinated universal time (UTC), with the first two being consecutive along the same orbit. Wave spectral inversions were performed from $2 \times 2.5 \mathrm{~km}$ blocks in the azimuth and range directions, respectively, corresponding to $512 \times 128$ SAR pixels, giving a wave spectrum every $2 \mathrm{~km}$.

Due to the analysis technique, the distance between the measurement point and the spectral estimation is at most $1.7 \mathrm{~km}$. In situ data reported here were obtained with four Datawell Waverider buoys (two are directional buoys, DW1 and DW4, while DW3 and DW2 measured heave only), one Nortek AWAC wavecurrent profiler, one Interocean S4DW wave-current meter, and a tripod (SA1) equipped with a Paroscientific pressure gauge and an EM MarshMcBirney current meter.

These instruments were deployed in the Golfe Normand-Breton, in the western English Channel, between Saint Malo and Granville, in water depths from 30 to $6 \mathrm{~m}$ (at high tide), with a typical tidal range of $10 \mathrm{~m}$ (Fig. 1). In spite of the limited area covered by each image, the maps of Hs obtained by the present algorithm appear to be rather smooth (Fig. 5 and 6). A detailed estimation of theoretical uncertainties on the SAR spectra and Hs estimates is beyond the scope of the present paper.

We now present comparisons of wave parameters obtained from the two images acquired in the morning of March 9 with incidence angles $31<\theta_{I}<37^{\circ}$ and the one acquired in the evening with incidence angles $19<\theta_{I}<26^{\circ}$. For reference, existing methods have only been used previously for imagettes acquired at $\theta_{I}=23^{\circ}$.

All images were acquired close to high tide, when currents are still relatively weak. At the time of the acquisitions, a weak to moderate southwesterly wind was blowing, but the sea state was dominated by large westerly swells propagating from the North Atlantic into the English Channel. Variations in space of the significant wave height $(\mathrm{Hs})$ and direction at the spectral peak $(\theta \mathrm{p})$ show the sheltering effects of islands, high shoals, and headlands, with reduced values of $\mathrm{Hs}$ and directions veering from westerly to southwesterly or northwesterly (e.g., to the East of the Island of Jersey, Figs. 3 and 4). These patterns closely resemble what can be obtained by numerical wave modeling. In particular, the wave height is reduced and the mean wave direction is seen to rotate downwave of the islands as a result of refraction and sheltering from the direct wave paths so that obliquely propagating waves are less attenuated. Crossing swells are found to the east of Jersey. Numerical simulations indicate that diffraction plays a negligible role for this kind of field conditions [14].

Figs. 5 and 6 only display wave parameters in image blocks that are not affected by land. Several criteria were tested to reject land effects, including an a priori land mask, a normalized image variance parameter $(\mathrm{NV})$, and the absolute value of the wave height Hs. The NV parameter is able to reject anomalous image blocks, but a proper threshold is difficult to define. A land mask such as the Global Self-consistent Hierarchical High-resolution Shoreline database [15] can be used to define land and proves useful for removing land-contaminated points close to the mainland and the Chausey archipelago. Yet, at high tide, the Minquiers shoals (Fig. 4) are fully under water and could be included in the wave analysis. Besides, abnormally large wave heights were found in the image row closest to the satellite track. Fig. 5 therefore includes all image blocks with $\mathrm{Hs}<6 \mathrm{~m}$ and a land fraction less than 0.05. In Fig. 6, we use $\mathrm{Hs}<6 \mathrm{~m}$ and a land fraction less than 0.07, which now includes the Minquiers shoals.

We now compare SAR-derived parameters to in situ data. Tables I and II summarize the main wave parameters: Hs, peak pe$\operatorname{riod} T_{\mathrm{p}}=1 / f_{\mathrm{p}}$ (estimated, in the case of SAR, from the peak wavelength), $\theta \mathrm{p}$, and directional spread at the peak of the spectrum $\sigma_{\theta \mathrm{p}}$. All these parameters can be obtained reliably from directional in situ instruments measuring surface displacements $(x, y, z)$ or bottom pressure and velocity $(p, u, v)[16]$.

As reported, SAR-derived spectra have a tendency to overestimate the wave heights by $10-70 \%$ in images acquired at lower incidence angles $\left(19-26^{\circ}\right)$. According to the inversion methodology, this is likely a result of an underestimation of the tilt MTF. The bias is strongly reduced for incidence angles larger than $30^{\circ}$, for which tilt modulation is weaker. Errors in the wave peak period Tp are also reported. Many likely sources of uncertainties besides the SAR inversion itself and/or the subimage sizes may be invoked, including errors on the dispersion relation between temporal (in situ) and spatial (SAR) wave spectra due to depth uncertainties and effect of currents (of the order $1 \mathrm{~m} / \mathrm{s}$ at the time of the image [17], [18]). Wave directions are generally well estimated, and directional spectra have a reasonably narrow swell shape.

However, at the shallowest site (SA1), a result spectrum shows a large component propagating against the observed swell direction. Such an artifact is due to the low SNR in the presence of very low winds that perturb the ambiguity removal process. For this particular subimage, the wave-induced backscatter intensity modulation is corrupted and a more dedicated filtering should be applied. In this case, the cross-correlation technique has difficulties resolving the propagation direction. A few other examples of wrong peak wave directions can be seen on Fig. 5 (e.g., at $48^{\circ} 54^{\prime} \mathrm{N}, 2^{\circ} 15^{\prime} \mathrm{W}$ ) and Fig. 6 (over the Minquiers shoals), where the waves are seen to propagate against the true wave direction. Again, these image blocks correspond to rather large values of the normalized variance and/or shallow water locations with short wavelength and occasionally strong wave breaking.

Full frequency-direction spectra were also estimated from in situ instruments using the maximum entropy method [19] and the SAR images. Above the in situ peak frequency, SAR results will often show spurious or misplaced secondary peaks [Fig. 3(a) and (b)] that may be related to both the nonlinear imaging process and the linear MTF. Wave directions were fairly well estimated with a low level of noise in directions away from the true directions [Fig. 5(c)]. For image blocks where the direction estimation breaks down, additional information on the likely wave direction (e.g., coming from offshore in coastal areas) may be used. 


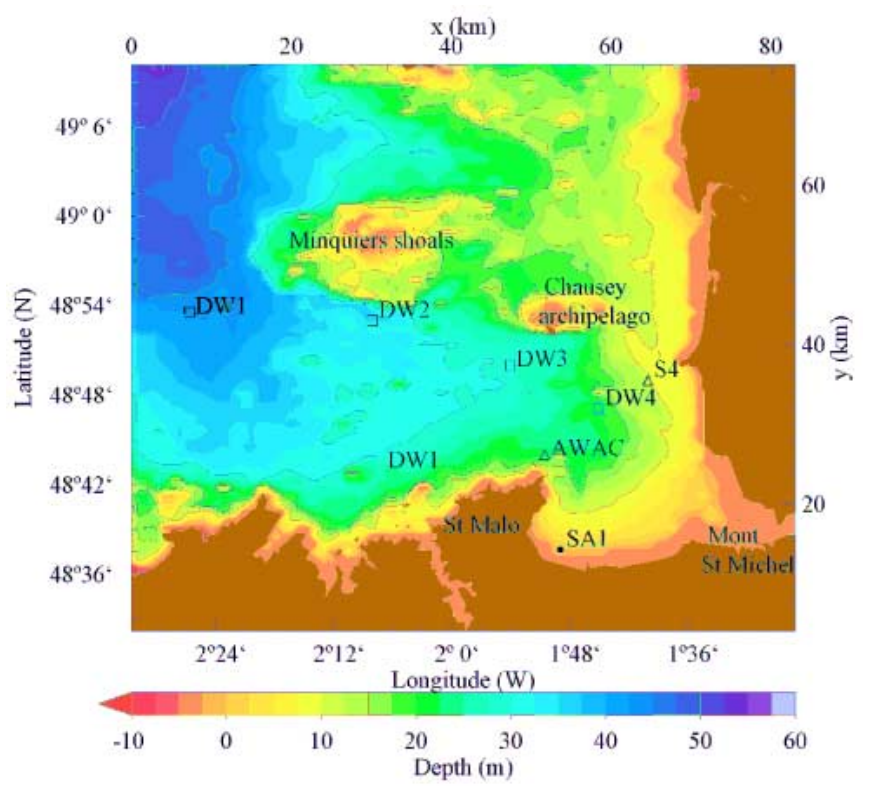

Fig. 4. Bathymetry and position of the in situ instruments. Depth is relative to the minimum astronomical tide.

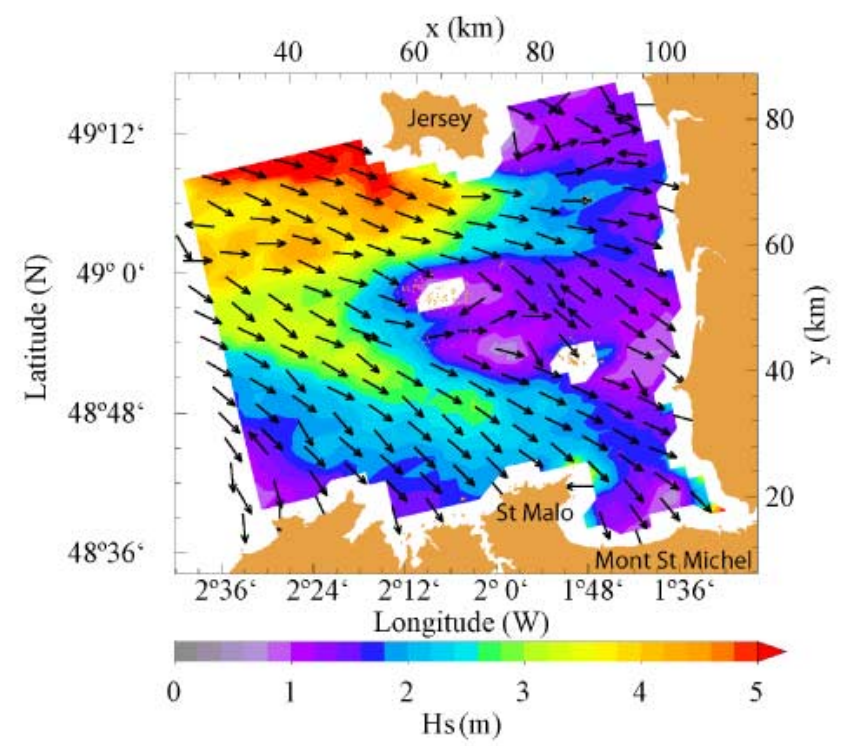

Fig. 5. SAR-derived significant wave height Hs (colors) and mean direction at the spectral peak $\theta \mathrm{p}$ (arrows) at $21 \mathrm{~h} 35 \mathrm{~min}$ UTC. The land mask based on [15] appears in brown. For clarity, only one out of the four arrows is shown.

\section{CONCLUSION}

The analysis of synthetic aperture radar (SAR) images can yield a large amount of information for coastal applications, including wave spectra and their variation in space. A straightforward adaptation of the ENVISAT advanced SAR (ASAR) level 2 algorithm to the processing of narrow swath complex images was described and demonstrated. As demonstrated with standard wave mode products, these SAR products contain phase information that was used to determine the wave propagation direction, without outside information. Thus, the instrument time integration is sufficient to provide useful changes between different looks at different incidence angles. Such a source of information is not included in conventional amplitude images for which a likely wave is probably sufficient. However, as for wave

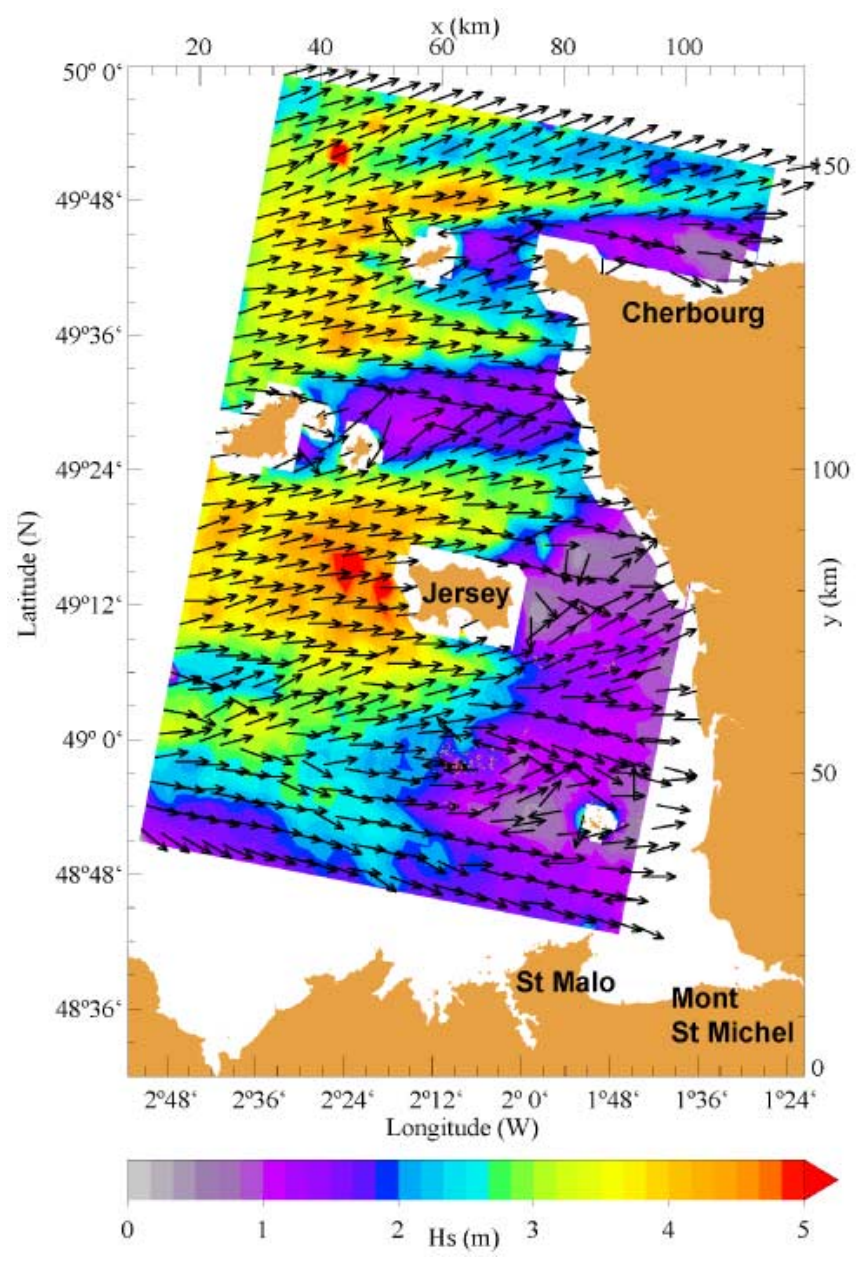

Fig. 6. SAR-derived $\mathrm{Hs}$ (colors) and $\theta \mathrm{p}$ (arrows) at $10 \mathrm{~h} 22 \mathrm{~min}$ to $10 \mathrm{~h} 23 \mathrm{~min}$ UTC. For clarity, only one out of the four arrows is shown.

mode products, speckle noise is strongly and simply reduced using the cross-spectral analysis of complex images. Comparisons with in situ data reveal an overall good description of swell 
TABLE I

MAIN WAVE PARAMETERS AT 10 h 22 min UTC (MARCH 9, 2003)

\begin{tabular}{lccccc}
\hline \multicolumn{1}{c}{ Site: } & DW1 & DW2 & DW3 & DW4 & AWA \\
\hline $\begin{array}{l}\text { Depth } \\
(\mathrm{m})\end{array}$ & 49 & 41 & 34 & 25 & 27 \\
true Hs & 2.35 & 0.90 & 1.14 & 1.08 & 1.23 \\
$\begin{array}{l}\text { SAR Hs } \\
(\mathrm{m})\end{array}$ & 2.38 & 0.93 & 0.99 & 0.92 & 1.62 \\
true Tp & 14.1 & 12.8 & 11.8 & 14.1 & 14.1 \\
$\begin{array}{l}\text { SAR Tp } \\
(\mathrm{s})\end{array}$ & 13.5 & 10.75 & 10.5 & 11.0 & 11.4 \\
true $\theta p$ & $292^{\circ}$ & N.A. & N.A. & $287^{\circ}$ & $300^{\circ}$ \\
$\begin{array}{l}\text { SAR } \theta p \\
(\text { deg) }\end{array}$ & $307^{\circ}$ & $276^{\circ}$ & $278^{\circ}$ & $280^{\circ}$ & $298^{\circ}$ \\
true $\sigma_{\theta p}$ & $13^{\circ}$ & N.A. & N.A. & $14^{\circ}$ & $26^{\circ}$ \\
$\begin{array}{l}\text { SAR } \sigma_{\theta p} \\
(\operatorname{deg})\end{array}$ & $15^{\circ}$ & $22^{\circ}$ & $41^{\circ}$ & $36^{\circ}$ & $25^{\circ}$ \\
\hline
\end{tabular}

TABLE II

Main WAVE PARAMETERS AT 21 h 44 min UTC (MARCH 9, 2003)

\begin{tabular}{lccccccc}
\hline \hline \multicolumn{1}{c}{ Site: } & DW1 & DW2 & DW3 & DW4 & AWA & S4 & SA1 \\
\hline $\begin{array}{l}\text { Depth } \\
(\mathrm{m})\end{array}$ & 49 & 41 & 34 & 25 & 27 & 15.1 & 5.51 \\
true Hs & 3.05 & 0.98 & 1.22 & 1.27 & 1.54 & 0.99 & 0.66 \\
$\begin{array}{l}\text { SAR Hs } \\
(\mathrm{m})\end{array}$ & 3.15 & 1.70 & 1.95 & 1.63 & 2.35 & 1.25 & 0.97 \\
true Tp & 15.2 & 12.8 & 12.8 & 14.3 & 16.4 & 14.3 & 13.0 \\
SAR Tp & 12.3 & 14.7 & 9.1 & 14.1 & 12.8 & 10.5 & 14.9 \\
$(\mathrm{~s})$ & & & & & & & \\
true $\theta \mathrm{p}$ & $294^{\circ}$ & N.A. & N.A. & $282^{\circ}$ & $305^{\circ}$ & $276^{\circ}$ & $14^{\circ}$ \\
$\begin{array}{l}\text { SAR } \theta p \\
(\operatorname{deg})\end{array}$ & $287^{\circ}$ & $283^{\circ}$ & $280^{\circ}$ & $286^{\circ}$ & $303^{\circ}$ & $270^{\circ}$ & $25^{\circ}$ \\
true $\sigma_{\theta p}$ & $13^{\circ}$ & N.A. & N.A. & $18^{\circ}$ & $24^{\circ}$ & $27^{\circ}$ & $5^{\circ}$ \\
$\begin{array}{l}\text { SAR } \sigma_{\theta p} \\
(\operatorname{deg})\end{array}$ & $7^{\circ}$ & $22^{\circ}$ & $31^{\circ}$ & $9^{\circ}$ & $23^{\circ}$ & $42^{\circ}$ & $39^{\circ}$ \\
\hline & & & & & & \\
\hline
\end{tabular}

propagation close to the coast. Frequency spectral shapes are found to be poorly represented at frequencies above twice the peak frequency. Overestimated wave heights for the lowest incidence angles indicate that the simplified CMOD-derived tilt function should be improved, especially for coastal areas. It should be noted that waves propagating in the radar azimuth direction were observed, with wavelength down to $60 \mathrm{~m}$. This is a very unusual situation due to a low wind speed and small wave slopes for which the cutoff is very weak. The imaging process for these waves is thus likely related to a RAR modulation mechanism such as described by [20] and not explicitly represented in the inversion algorithm used here.

Although the estimation of wave height is intrinsically limited by random wave variations on the scale of wave groups, a relatively low level of noise was found in SAR-derived maps of significant wave height at a resolution of $2 \mathrm{~km}$. This is certainly encouraging for even higher resolution processing possibly based on a combination with simple ray-tracing models [14].

\section{APPENDIX \\ SAR RESAMPLING}

We propose a new method for removing nonlinear SAR mapping distortions when look-up tables based on nonlinear simulations of the direct SAR transform are not available for all possible incidence angles, water depths, and wind speeds.
SAR processing uses the measured Doppler shifts in the returned radar signal to determine the position of the scatters in the azimuth (along-track) direction. If a random phase fluctuation is introduced in the phase of each individual echo, the echo will then be misplaced at a position $r+\xi$ instead of its true position $r$. Following such a description, the SAR image intensity $I_{\mathrm{SAR}}$, at a given horizontal location $r$ and time $t$, will result from a stochastic redistribution of the RAR intensity $I_{\mathrm{RAR}}$ at $r^{\prime}$ as

$$
\begin{aligned}
& I_{\mathrm{SAR}}(r, t) \\
& \quad=\int_{r^{\prime}} I_{\mathrm{RAR}}\left(r^{\prime}, t\right) h\left(r-r^{\prime}-\xi\left(r^{\prime}, t\right)\right) \mathrm{d} r^{\prime} \\
& \quad=h *\left[\int_{r^{\prime}} I_{\mathrm{RAR}}\left(r^{\prime}, t\right) \delta\left(r-r^{\prime}-\xi\left(r^{\prime}, t\right)\right) \mathrm{d} r^{\prime}\right] \\
& \quad=\sum_{r^{\prime \prime}} h\left(r-r^{\prime \prime}\right) \sum_{r^{\prime}}\left\{\left|\frac{\mathrm{d} r^{\prime}}{\mathrm{d} r}\right| I_{\mathrm{RAR}}(r, t)\right\}_{r^{\prime \prime}=r^{\prime}+\xi\left(r^{\prime}, t\right)},
\end{aligned}
$$

where $h$ is the instrumental impulse response and $\left|\mathrm{d} r^{\prime} / \mathrm{d} r\right|$ is the Jacobian representing the local variation of scatter density. The mean intensity is conserved with this mapping between $I_{\mathrm{RAR}}$ and $I_{\mathrm{SAR}}$, so that after processing, a high-resolution SAR image can thus still be used as a scatter meter to restitute wind information from the intensity averaged over a large enough area. However, after random redistribution, the SAR intensity pixel covariance function will certainly not coincide with the expected RAR intensity pixel covariance function $\left\langle I_{\mathrm{RAR}}(r) I_{\mathrm{RAR}}\left(r^{\prime}\right)\right\rangle$, where angular brackets denote expected values.

Considering the RAR covariance function for small lags $\varepsilon$, $\left\langle I_{\mathrm{RAR}}(r) I_{\mathrm{RAR}}(r+\varepsilon)\right\rangle$, and applying SAR random mapping, this function will become approximately equal to $\left\langle I_{\mathrm{SAR}}(r+\right.$ $\left.\xi(r)) I_{\mathrm{SAR}}(r+\varepsilon+\xi(r+\varepsilon))\right\rangle$ for RAR covariance functions that decay fast with increasing $\varepsilon$. Assuming spatial homogeneity, the RAR pixel covariance function around its origin (for $r=0$ ) is mapped as $\left\langle I_{\mathrm{SAR}}(\xi(0)) I_{\mathrm{SAR}}(\varepsilon+\xi(\varepsilon))\right\rangle$ in the SAR domain. The initial lag is now a random variable, increased in the mean by a factor $\left\{1+\left\langle[\xi(\varepsilon)-\xi(0)]^{2}\right\rangle / \varepsilon^{2}\right\}^{1 / 2}$. In the case of Gaussian statistics for a random displacement $\xi$, the mean lag increase factor depends upon the displacement covariance function $\rho_{\xi}$ around its origin. For sufficiently small initial lags $\varepsilon$, SAR mapping will be characterized by the coefficient $\left(1+\partial^{2} \rho_{\xi} / \partial r^{2}\right)$ [6]. Thus, the SAR image covariance function measured at a small spatial lag $\Delta r$ corresponds to a RAR covariance function evaluated at a smaller spatial lag $\delta r$. The SAR covariance function indeed exhibits very slow decays in the along-track direction, corresponding to the very fast decay in the spectral domain (cutoff).

At small spatial lags, a resampling operation can be applied to correct for most of the SAR mapping distortion. This resampling operation is dominated by the second derivative of the mean squared displacement variance at the origin $\partial^{2} \rho_{\xi} / \partial r^{2}(0)$. This parameter is dominated by the smallest scales and can be shown to saturate with increasing wind speeds. On the contrary, for larger spatial lags $r_{\mathrm{L}}$, the SAR and linearly modulated RAR covariance functions will almost coincide. This shall occur when the displacement variance is small compared to the spatial lag, i.e., $\rho_{\xi}(0) \ll r_{\mathrm{L}}^{2}$. At these larger scales, the imaging process is almost linear and there is no need to resample the observed 
SAR covariance function. This effect is therefore local and not periodic, and should not be deconvoluted in the Fourier domain.

Considering the transformation, $\delta r \rightarrow \Delta r$ with $\Delta r$ as the SAR image azimuthal resolution, and an a priori Gaussian shape for the displacement covariance function, the theory gives

$$
\delta r^{2}+2 \rho_{\xi}(0)\left[1-\exp \left(-\frac{\delta r^{2}}{\mathrm{~L}^{2}}\right)\right]=\Delta r
$$

with $L$ as the random displacement correlation length, which was found to be proportional to $\rho_{\xi}(0)$ for all wind speeds. The control parameter is thus $\rho_{\xi}(0)$, which is related to the measurable cutoff parameter [Fig. 2(f)] [21]. The resampling applied in the present paper is a simple linear reduction in the cross-covariance function. The image cross-covariance function is divided by an estimation of the nonlinear remapping function using a Hilbert transform, which only affects the short lags [Fig. 2(d) and (e)]. Detailed expressions for this are rather cumbersome.

\section{ACKNOWLEDGMENT}

In situ data were acquired thanks to the contribution of CETMEF and the dedication of the crew and divers from the R/V Laplace and Mission Hydrographique de l'Atlantique. Cruise planning and in situ data processing were done by F.A. with the help of D. Corman. The authors are thankful to the European Space Agency (ESA) for providing ENVISAT images and P. Le Hir for the SA1 in situ measurements. The resampling technique was designed by F.C. and B. C., and implemented by F. C. Images were analyzed by F. C. SAR images were provided by the ESA.

\section{REFERENCES}

[1] P. Janssen, The Interaction of Ocean Waves and Wind. Cambridge, U.K.: Cambridge Univ. Press, 2004, p. 300.

[2] W. E. Rogers, P. A. Hwang, and D. W. Wang, "Investigation of wave growth and decay in the SWAN model: three regional-scale applications," J. Phys. Oceanogr., vol. 33, no. 2, pp. 366-389, Feb. 2003.

[3] L. Aouf, J. M. Lefevre, D. Hauser, and B. Chapron, "Impact of ASAR ENVISAT directional wave spectra on wave forecast," in Int. Geoscience Remote Sensing Symp. (IGARSS), Toulouse, France, Jul. 21-25, 2003, pp. 3178-3180.

[4] F. Ardhuin, T. H. C. Herbers, W. C. O'Reilly, and P. F. Jessen, "Swell transformation across the continental shelf part II: validation of a spectral energy balance equation," J. Phys. Oceanogr., vol. 33, no. 9, pp. 1940-1953, Sep. 2001.

[5] H. L. Tolman, "A third generation model for wind on slowly varying, unsteady and inhomogeneous depth and currents," J. Phys. Oceanogr., vol. 21, no. 6, pp. 782-797, Jun. 1991.

[6] H. E. Krogstad, "A simple derivation of Hasselmann's nonlinear ocean-synthetic aperture radar transform," J. Geophys. Res., vol. 97, no. C2, pp. 2421-2425, Feb. 1992.

[7] V. Kudryavtsev, D. Hauser, G. Caudal, and B. Chapron, "A semiempirical model of the normalized radar cross-section of the sea surface 2 . Radar modulation transfer function," J. Geophys. Res., vol. 108, no. C3, p. 8055 , Mar. 2003.

[8] K. Hasselmann and S. Hasselmann, "On the nonlinear mapping of an ocean wave spectrum into a synthetic aperture radar image spectrum and its inversion," J. Geophys. Res., vol. 96, no. C6, pp. 10713-10729, Jun. 1991.

[9] C. Brüning, S. Hasselmann, and K. Hasselmann, "First evaluation of ERS-1 synthetic aperture radar wave mode data," Global Atmos. Ocean Syst., vol. 2, no. 1, pp. 61-98, 1994.

[10] S. Hasselmann, C. Brüning, K. Hasselmann, and P. Heimbach, "An improved algorithm for the retrieval of ocean wave spectra from SAR image spectra," J. Geophys. Res., vol. 101, no. C7, pp. 16615-16629, Jul. 1996.

[11] C. Mastenbroek and C. F. de Valk, "A semi-parametric algorithm to retrieve ocean wave spectra from SAR," J. Geophys. Res., vol. 105, no. C2, p. 3947, Feb. 2000
[12] G. Engen and H. Johnsen, "SAR-Ocean wave inversion using image cross spectra," IEEE Trans. Geophys. Remote Sensing, vol. 33, no. 4, pp. 1047-1056, Jul. 1995.

[13] Y. Quilfen, B. Chapron, T. Elfouhaily, K. Katsaros, and J. Tournadre, "Observation of tropical cyclones by high-resolution scatterometry," $J$. Geophys. Res., vol. 103, no. C4, pp. 7767-7786, Apr. 1998.

[14] W. C. O'Reilly and R. T. Guza, "A comparison of two spectral wave models in the Southern California Bight," Coast. Eng., vol. 19, pp. 263-282, 1993.

[15] P. Wessel and W. H. F. Smith, "A global self-consistent, hierarchical, high-resolution shoreline database," J. Geophys. Res., vol. 101, no. B4, pp. 8741-8743, Apr. 1996.

[16] A. J. Kuik, G. P. van Vledder, and L. H. Holthuijsen, "A method for the routine analysis of pitch-and-roll buoy wave data," J. Phys. Oceanogr. vol. 18, no. 7, pp. 1020-1034, Jul. 1988.

[17] B. Chapron, F. Collard, and F. Ardhuin, "Direct measurements of ocean surface velocity from space: interpretation and validation," J. Geophys. Res., vol. 11, no. C7, Jul. 2005.

[18] F. Ardhuin, A. D. Jenkins, D. Hauser, A. Reniers, and B. Chapron, "Waves and operational oceanography: towards a coherent description of the upper ocean for applications," Eos, vol. 86, no. 4, pp. 37-39, Jan. 2005.

[19] A. Lygre and H. E. Krogstad, "Maximum entropy estimation of the directional distribution in ocean wave spectra," J. Phys. Oceanogr, vol. 16, no. 12, pp. 2052-2060, Dec. 1986.

[20] F. Askari and W. C. Keller, "Real aperture radar imaging of ocean waves during SAXON-FPN: a case of azimuth-travelling waves," J. Geophys. Res., vol. 99, no. C5, pp. 9817-9833, May 1994.

[21] V. Kerbaol, B. Chapron, and P. W. Vachon, "Analysis of ERS 1/2 synthetic aperture radar wave mode imagettes," J. Geophys. Res., vol. 103, no. C4, pp. 7833-7846, Apr. 1998.

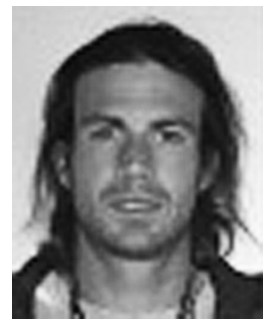

Fabrice Collard was born in 1973. He graduated with a degree in off-shore engineering from Ecole Centrale de Lyon, France, in 1996, and received the Ph.D. degree in oceanography and meteorology environment. His thesis was dedicated to the three-dimensional (3-D) aspect of wind-wave field.

He spent 2 years working on HF radars as a PostDoctoral Research Associate at RSMAS, Miami. He is currently working at Brest Operational Observation Systems and Technologies, BOOST Technologies, Brest, France, on the validation of the synthetic aperture radar of ENVISAT (http://www.boost-technologies.com).

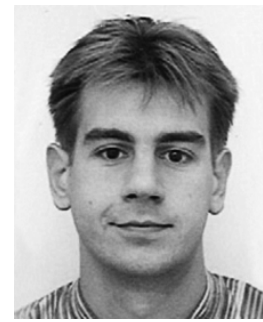

Fabrice Ardhuin was born in 1975. He graduated with a degree in fluid mechanics and geophysics from Ecole Polytechnique, Palaiseau, France, in 1997 and received the Ph.D. degree in oceanography at the U.S. Naval Postgraduate School, Monterey, CA, in 2001, with a thesis dedicated to the transformation of waves across the continental shelf.

In 2001, he joined the French Navy Hydrographic and Oceanographic Service (SHOM), Brest, France, as a Researcher/Assistant Manager of military oceanography programs. His main research interests are related to operational oceanography, in particular, wave forecasting and the combination of wave, currents, and remote sensing (http://surfouest.free.fr/ WOO2003; http://www.shom.fr/fr_page/fr_act_oceano/vagues/vagues.htm).

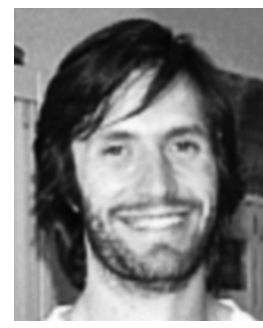

Bertrand Chapron was born in 1962. He graduated from the Institut National Polytechnique de Grenoble, France, in 1984 and received the Doctorat National (Ph.D.) degree in fluid mechanics in 1988.

He spent 3 years as a Post-Doctoral Research Associate at the NASA/GSFC/Wallops Flight Facility, USA. He has experience in applied mathematics, physical oceanography, electromagnetic waves theory, and its application to ocean remote sensing. He is currently the Head of Oceanography from Space Laboratory, IFREMER, Brest, France 